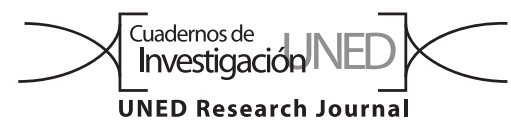

\title{
Fitotoxicidad de compost producido con cultivos de microorganismos de montaña y lodos de biodigestor
}

\author{
Fabricio Camacho Céspedes ${ }^{1}$, Lidieth Uribe Lorío ${ }^{2}$, Quint Newcomer ${ }^{3}$, Karen Masters ${ }^{4}$ \& Maureen Kinyua ${ }^{5}$ \\ 1. University of Georgia, Costa Rica Campus. Apartado 108-5655, Monteverde, Costa Rica; fabricio@uga.edu, fabricio.camacho.cr@gmail.com \\ 2. Universidad de Costa Rica, Centro de Investigaciones Agronómicas; lidieth.uribe@ucr.ac.cr \\ 3. University of Georgia, Odum School of Ecology; quintcostarica@gmail.com \\ 4. Council on International Educational Exchange, Sustainability Center Monteverde, Costa Rica; kmasters@ciee.org \\ 5. University of California Davis, Department of Civil and Environmental Engineering; mnkinyua@ucdavis.edu
}

Recibido 11-VII-2018 • Corregido 21-IX-2018 • Aceptado 23-X-2018

\begin{abstract}
Phytotoxicity of compost produced with mountain microorganisms and biodigester sludge". Introduction: compost quality may be enhanced with compost optimization agents. These agents must guarantee safety. Compost phytotoxicity tests are an economic, rapid and reliable mechanism to detect toxic substances in compost that may inhibit seed germination and plant growth. Objective: to evaluate the phytotoxicity of mountain microorganisms and biodigester sludge as compost optimization agents Methods: complete randomized blocks bioassay with cucumber seeds (Cucumis sativus). Results: individual use of these agents has no inhibitory effects in cucumber germination and growth and is thus safe. However, added simultaneously, they have inhibitory effects on germination and growth of cucumber, possibly from the phytotoxic effect of excess Zinc and Boron. Conclusion: we recommend caution and further tests with additional growth stages and species.
\end{abstract}

Key words: quality of organic fertilizer, sustainable agriculture, biofertilization, nutrient cycling.
RESUMEN: Introducción: la calidad del compost puede ser ajustada a través de la incorporación de agentes optimizadores. La selección de los agentes debe hacerse en forma cuidadosa para efectos de garantizar la seguridad del compost maduro. Las pruebas de fitotoxicidad son un mecanismo económico, rápido y confiable para detectar en el compost la presencia de sustancias tóxicas inhibidoras de la germinación y el crecimiento de cultivos. Objetivo: evaluar la fitotoxicidad de los microorganismos de montaña y los lodos de biodigestor como agentes optimizadores del compost. Métodos: bioensayo de germinación y crecimiento de semillas de pepino (Cucumis sativus) utilizando un arreglo de bloques completamente aleatorizados. Resultados: el empleo individual de estos agentes, no tiene un efecto inhibidor en la germinación y el crecimiento del pepino, por lo que pueden ser considerados como materiales seguros para la optimización de la calidad del compost. Sin embargo, la incorporación simultánea de ambos agentes en la producción de este abono orgánico provocó una disminución significativa en la germinación y el crecimiento inicial del pepino. Este resultado podría explicarse por el efecto fitotóxico que ejerce el exceso de Zinc y Boro detectado en el compost que combina ambos materiales. Conclusión: recomendamos utilizar este compost con precaución y llevar a cabo otras pruebas de crecimiento para identificar si la fitotoxicidad observada se mantiene sobre otros cultivos y sobre otras etapas de desarrollo más avanzadas a la germinación y el crecimiento inicial.

Palabras clave: calidad de abono orgánico, agricultura sostenible, biofertilización, reciclaje de nutrientes.
El compostaje es una tecnología apropiada para el manejo de los residuos orgánicos, al ser un proceso donde se transforma eficientemente este tipo de materiales en compuestos estables que pueden ser reincorporados en los sistemas de producción agrícola para el mejoramiento de la fertilidad natural del suelo (Haug, 1993; Beltrán et al., 2002; Golabi, Denney, \& lyekar., 2007; Diaz, Bertoldi, \& Bidlingmaier, 2011; FAO, 2013; Lazcano et al., 2014). El compost resultante de dicho proceso de conversión contiene sustancias nutritivas para los cultivos (Paulin \& O'Malley, 2008; FAO, 2013), y puede hacer un aporte significativo de agua, microorganismos y carbono en el suelo (Bot \& Benites, 2005; Lazcano et al., 2014).

El compost es un fertilizante de próxima generación cuya calidad requiere ser afinada con el fin de ampliar su potencial como herramienta para lograr el avance hacia la intensificación sostenible y la de-carbonización de la 
agricultura (Kotschi, 2015; Lehner \& Rosenberg, 2017). La calidad del compost depende en gran medida de las características de los materiales que se emplean en la producción (Campitelli \& Ceppi, 2008; Boldrin et al., 2009; Bernal et al., 2017), por lo que dicha calidad puede ser ajustada a través del empleo de agentes optimizadores que permitan aumentar la concentración de nutrientes, así como la cantidad de materia orgánica, la capacidad de retención de humedad y el potencial para el almacenaje de carbono del producto terminado.

La selección de agentes optimizadores de la calidad del compost debe implementarse a través de un proceso cuidadoso y ordenado. Estos materiales deben ser abundantes, económicos y fáciles de conseguir. Idealmente deben tener el potencial de favorecer el reciclaje de nutrientes $u$ otros elementos escasos de los sistemas productivos, propiciar el manejo de residuos y aportar en el mejoramiento de una o varias de las características del compost maduro. De igual forma, es necesario asegurar que dichos materiales estén libres de sustancias fitotóxicas (Cohen, Flint, \& Hines, 2009) que puedan afectar el desarrollo de los cultivos, lo cual puede ser evaluado en forma confiable, rápida y económica, a través de la implementación de ensayos de germinación y crecimiento. Estos ensayos consisten en medir la respuesta de un cultivo sensible ante la exposición a un compost experimental (que contiene los agentes optimizadores) y a un control (Helfrich et al., 1998). Las diferencias en el desarrollo del cultivo, aporta evidencia sobre la existencia de sustancias fitotóxicas en el compost valorado (Szabó, 2000; Stoffella \& Kahn, 2001; Reigosa \& Pazos-Malvido, 2007). El presente trabajo tuvo como objetivo evaluar en condiciones in vitro la fitotoxicidad de microorganismos de montaña (MM) y lodos digeridos de biodigestor (LDBIO) como agentes optimizadores del compost.

\section{MATERIALES Y MÉTODOS}

Preparación de los compost con microrganismos
de montaña y lodos de biodigestor: se prepararon seis diferentes tipos de compost (ver Cuadro 1) siguiendo la metodología de FAO (2013), para los cuales se utilizó en cada uno de ellos una mezcla base de $460 \mathrm{~kg}$ de biomasa fresca compuesta de $200 \mathrm{~kg}$ de residuos de cosecha de hortalizas, legumbres y frutales, $200 \mathrm{~kg}$ de hojas de banano y $60 \mathrm{~kg}$ de astillas de madero negro (Gliricidia sepium (Jacq.) Kunth), procurando una relación C:N de 30:1 a 35:1. Los compost fueron elaborados en la Finca Experimental para la Agricultura Sostenible de la Universidad de Georgia, ubicada en San Luis de Monteverde, Puntarenas, Costa Rica, $10^{\circ} 16^{\prime} 58,38^{\prime \prime} \mathrm{N}$ \& $84^{\circ} 47^{\prime} 52,93^{\prime \prime} \mathrm{W}$ a $1100 \mathrm{msnm}$, durante octubre del 2016 a enero del 2017.

\section{CUADRO 1}

Composición de los compost elaborados para evaluar la fitotoxicidad de los microorganismos de montaña y los lodos digeridos de biodigestor como agentes optimizadores de compost, 2017

\footnotetext{
Nombre Descripción del compost

COPURO Compost puro: mezcla base más $10 \mathrm{~kg}$ de agua al inicio de la elaboración del compost y un promedio de $0,8 \mathrm{~kg} \mathrm{H}_{2} \mathrm{O} / \mathrm{día}$ durante la etapa de estabilización.

COMMR Compost con MM: mezcla base más 10kg de MMR líquido activado al inicio de la elaboración del compost y un promedio de 0,8kg MMR/día durante la etapa de estabilización. La R significa que el cultivo de MM lleva todos los compuestos citados en la metodología de elaboración, incluyendo el inóculo del suelo del bosque.

COMMP Compost con MM placebo: mezcla base más $10 \mathrm{~kg}$ de MMP líquido activado aplicado al inicio de la elaboración del compost y un promedio de $0,8 \mathrm{~kg}$ MMP/día durante la etapa de estabilización. La P significa que el cultivo de MM lleva todos los compuestos citados en la metodología de elaboración, excepto el inóculo del suelo del bosque, para discriminar que el efecto optimizador esté realmente asociado a la incorporación de los microorganismos en el cultivo.

COLDB Compost con LDBIO: mezcla base más 40kg de LDBIO, 10kg de agua al inicio de la elaboración del compost y un promedio de $0,8 \mathrm{~kg} \mathrm{H}_{2} \mathrm{O}$ /día durante la etapa de estabilización.

COMMR Compost con MM y LDBIO A: mezcla base más 40kg de LDBIO, $10 \mathrm{~kg}$ de MMR líquido activado al inicio de la LDBA elaboración del compost y un promedio de 0,8kg MMR /día durante la etapa de estabilización.

COMMR Compost con MM y LDBIO B: Compost puro elaborado con 200kg de residuos de brosa de café, 200kg de hojas de LDBB banano y $60 \mathrm{~kg}$ de astillas de madero negro, $40 \mathrm{~kg}$ de LDBIO, $10 \mathrm{~kg}$ de MMR líquido activado al inicio de la elaboración del compost y un promedio de 0,8kg MMR/día durante la etapa de estabilización.
} 
CUADRO 2

Composición de los tratamientos elaborados para evaluar la fitotoxicidad del compost optimizado con microorganismos de montaña y lodos digeridos de biodigestor, 2017

\begin{tabular}{|c|c|c|c|}
\hline T\# & Nombre & Categoría & Descripción del tratamiento (volumen) \\
\hline T1 & COPURO & Control & Mezcla de 33\% COPURO +67\% Suelo \\
\hline $\mathrm{T} 2$ & COMMR & Experimental & Mezcla de $33 \%$ COMMR +67\% Suelo \\
\hline T3 & СOMMP & Experimental & Mezcla de $33 \%$ COMMP $+67 \%$ Suelo \\
\hline $\mathrm{T} 4$ & COLDB & Experimental & Mezcla de 33\% COLDB +67\% Suelo \\
\hline T5 & COMMRLDBA & Experimental & Mezcla de $33 \%$ COMMRLDBA $+67 \%$ Suelo \\
\hline T6 & COMMRLDBB & Experimental & Mezcla 33\% COMMRLDBB +67\% Suelo \\
\hline
\end{tabular}

Los microorganismos de montaña fueron producidos siguiendo la metodología de Suchini Ramírez (2012) utilizando hojarasca y humus proveniente del bosque de la Reserva Forestal del Campus de la Universidad de Georgia. Los lodos fueron extraídos de uno de los biodigestores tubulares de flujo continuo de la misma finca experimental, el cual es alimentado con excretas porcinas en suspensión 1:4; estos tuvieron un periodo de acumulación de cinco años en el reactor.

Evaluación de la fitotoxicidad: la evaluación de la fitotoxicidad de los agentes optimizadores del compost se llevó a cabo a través de un ensayo de germinación y crecimiento con semillas de pepino (Cucumis sativus), en el cual se utilizó un diseño experimental de bloques completos al azar (Hernández, Fernandez, \& Baptista, 2010) con seis tratamientos, seis repeticiones y un $n=90$ semillas por tratamiento (Cuadro 2). Se utilizó el pepino como especie indicadora debido a la alta sensibilidad de esta planta a sustancias tóxicas presentes en el sustrato de germinación (Wang \& Keturi, 1990). El estudio fue realizado en el Laboratorio de Microbiología Agrícola del Centro para Investigaciones Agronómicas de la Universidad de Costa Rica.

Las semillas de pepino fueron colocadas en platos petri con una mezcla de $67 \%$ del volumen de suelo tamizado y $33 \%$ de compost. A cada plato se le aplicó $5 \mathrm{~mL}$ de agua destilada con una bomba de aspersión manual para favorecer la germinación. Los platos fueron forrados con papel craft y colocados en un espacio oscuro a temperatura ambiente por siete días. Al séptimo día, los platos fueron abiertos en el mismo orden en que fueron guardados. A cada semilla se le registró la longitud total de la raíz y del tallo en $\mathrm{cm}$ con una regla milimétrica y el peso fresco total con una balanza electrónica calibrada a $0,00 \mathrm{~g}$.

Los datos de germinación y crecimiento fueron incorporados en una matriz de Excel, y ordenados según el tipo de tratamiento, a partir de los cuales se calculó el Porcentaje Relativo de Semillas Germinadas (PRSG\%), el Crecimiento Relativo Radicular (CRR\%), el Índice de Germinación (IG\%) y el Índice de Crecimiento (IC\%), utilizando el método adaptado de Pampuro et al. (2017) de la siguiente forma:

PRSG (\%): ( $\mathrm{n}$ de semillas germinadas en compost experimental / $\mathrm{n}$ de semillas germinadas en control) $X 100$

CRR (\%): (promedio longitud radicular en compost experimental / promedio longitud radicular en control) $\mathrm{X} 100$

IG (\%): (PRSG X CRR) / 100

IC (\%): (peso promedio en compost experimental / peso promedio en control) $\times 100$

Análisis estadísticos: se utilizaron las pruebas Kruskal-Wallis y Wilcoxon para identificar las diferencias entre los tratamientos experimentales y el control con respecto PRSG\%, CRR\%, IG\% y IC\%. Ambos análisis fueron realizados con el software analítico $\mathrm{R}$ versión 3.4.1win (R Core Team, 2018).

Ética, conflicto de intereses y declaración de financiamiento: los autores declaran haber cumplido con todos los requisitos éticos y legales pertinentes, tanto durante el estudio como en el manuscrito; que no hay conflictos de interés de ningún tipo, y que todas las fuentes financieras se detallan plena y claramente en la sección de agradecimientos. Asimismo, están de acuerdo con la versión editada final del documento. El respectivo 
documento legal firmado se encuentra en los archivos de la revista.

\section{RESULTADOS}

Proceso de elaboración del compost: no se detectaron anomalías en el proceso de compostaje con la incorporación de microorganismos y lodos como agentes optimizadores. Todas las pilas de compost experimentaron las tres etapas teóricas del compostaje durante el proceso de producción: mesófila, termófila y mesófila dos. El tiempo aproximado de estabilización fue de $70 \mathrm{~d}$. en todas las pilas. A partir de ese periodo, los seis tipos de compost producido entraron en un lapso de maduración. Este periodo tuvo una duración aproximada de 75d.

Evaluación de los niveles de fitotoxicidad: las pruebas de Kruskal Wallis revelan que existen diferencias significativas $(p<0,05)$ entre todos los tratamientos para los índices calculados (PRSG\%, CRR\%, IG\%, IC\%) (Cuadro 3), lo cual indica que la adición de microorganismos y lodos tiene una influencia perceptible sobre la germinación y el crecimiento inicial de las semillas de pepino (Cucumis sativus) expuestas a los diferentes tipos de abono.

Porcentaje Relativo de Semillas Germinadas (PRSG\%): la prueba pareada de Wilcoxon revela que los porcentajes de germinación promedio de los tratamientos T2, T3 y T4 no difieren significativamente al control $(p>0,05)$ (Fig. 1). Mientras que el PRSG \% promedio de los tratamientos T5 y T6 son significativamente menores al control $(p<0,05)$. Esto indica que el empleo integrado de microorganismos y lodos como agentes optimizadores del compost en las concentraciones utilizadas, tiene un efecto inhibidor en la germinación de las semillas de pepino, y podría inferirse que el empleo integrado de estos compuestos podría tener un efecto fitotóxico sobre cultivos sensibles.

Crecimiento Relativo Radicular (CRR\%): la prueba de Wilcoxon indica que el CRR\% promedio del T2 (COMMR) es significativamente mayor al control $(p<0,05)$ (Fig. 2), por lo que se podría inferir que la aplicación de compost tratado con microorganismos tiene efecto estimulador en el crecimiento radicular del cultivo experimental (Pampuro et al., 2017). El CCR\% promedio de los tratamientos T3 y T4 no difieren con respecto al control ( $p>0,05)$, mientras que el de los tratamientos T5 y T6 es significativamente menor al T1 $(p<0,05)$. Estos resultados aportan evidencia de que el empleo integrado de microorganismos y lodos como agentes optimizadores del compost en las concentraciones utilizadas, tiene un efecto inhibidor en desarrollo radicular del cultivo de prueba, y podría inferirse que el empleo integrado de estos compuestos podría tener un efecto fitotóxico sobre cultivos sensibles.

Î́ndice de Germinación (IG\%): la prueba de Wilcoxon muestra que el IG\% promedio de los tratamientos T2,T3 y T4 no difieren significativamente del control (T1) $(\mathrm{p}>0,05)$ (Fig. 3). Estos tratamientos presentan un IG\%>80\%, por lo que de acuerdo el criterio de Zucconi (1981), estos tres tipos de compost están libres de fitotoxinas. El

CUADRO 3

Promedios de los diferentes índices y estadísticos calculados para evaluar la fitotoxicidad de los microorganismos de montaña y los lodos digeridos de biodigestor como enmiendas optimizadoras del compost, 2017

\begin{tabular}{|c|c|c|c|c|}
\hline Tratamiento & PRSG\% & CRR\% & IG\% & $\mathrm{IC} \%$ \\
\hline T1 COPURO (Control) & $100,00(a)$ & $100,00(a)$ & $100,00(a)$ & $100,00(a)$ \\
\hline T2 COMMR & $95,11(\mathrm{a})$ & $128,90(b)$ & $131,55(a)$ & $115,77(a)$ \\
\hline Т3 СОММР & $109,97(a)$ & $105,52(a)$ & $113,86(a)$ & $99,31(a)$ \\
\hline T4 COLDBIO & $93,70(a)$ & $102,24(a)$ & $106,53(a)$ & $84,18(a)$ \\
\hline T5 COMMRLDBA & $57,98(b)$ & $45,00(b)$ & $28,69(b)$ & $44,21(b)$ \\
\hline T6 COMMRLDBB & $65,12(b)$ & 77,35 (b) & $53,25(b)$ & 57,96 (b) \\
\hline Kruskal-Wallis chi-square & 14,40 & 16,24 & 16,42 & 11,47 \\
\hline Valor de p para Kruskal-Wallis & 0,013 & 0,006 & 0,005 & 0,04 \\
\hline Notas & NA & $\Psi$ & $\mathrm{F}$ & $€$ \\
\hline
\end{tabular}

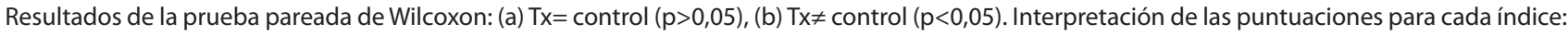
$\Psi>100 \%=$ existe efecto estimulador del crecimiento radicular (Pampuro et al., 2017). F $>80 \%=$ compost libre de fitotoxinas (Zucconi, 1981). $€>100 \%$ = compost libre de fitotoxinas (Piemonte, 2002). 


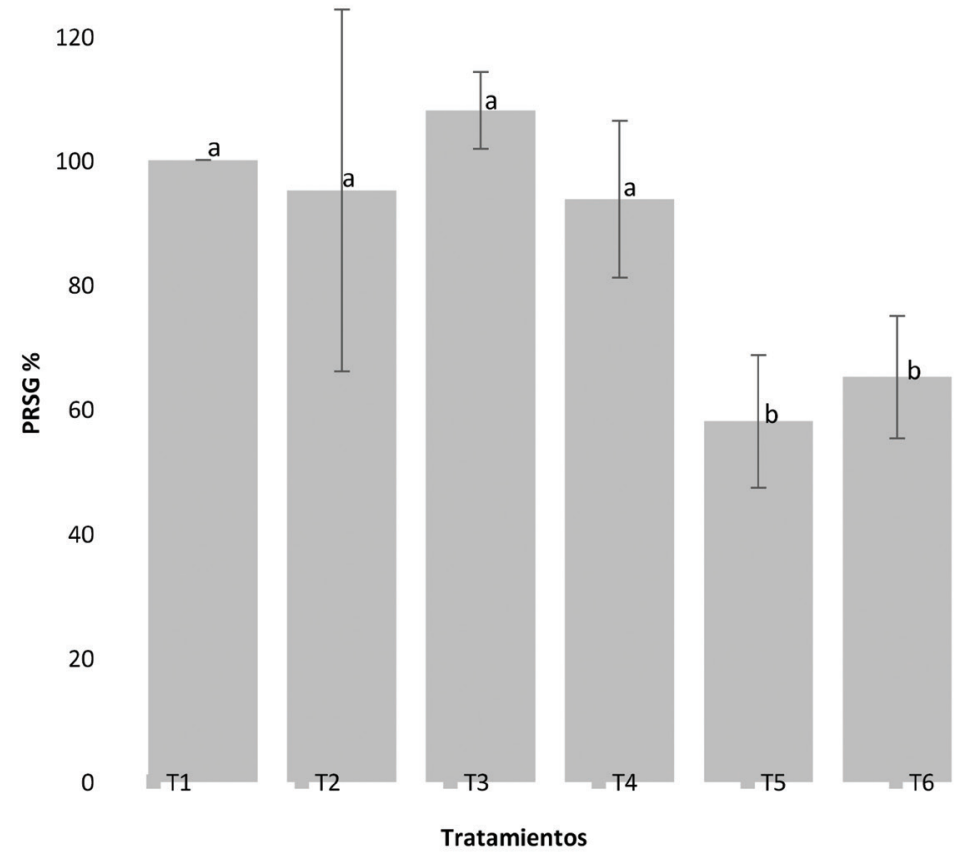

Fig. 1. Porcentaje Relativo de Semillas Germinadas (PRSG\%) de pepino (Cucumis sativus) expuestas a T1 (compost puro sin MM o LDBIO), T2 (compost COMMR), T3 (compost COMMP), T4 (compost COLDBIO), T5 (compost COMMLDBIOA), T6 (compost COMMLDBIOB). Promedio de los bloques con sus respectivos errores estándar. (a) Tx= control $(p>0,05),(b) T x \neq c o n t r o l(p<0,05)$. Existen diferencias significativas entre el T5 y T6 con respecto al T1 control (Wilcoxon, p<0,05). El empleo integrado de MM+LDBIO podría tener un efecto fitotóxico sobre cultivos sensibles.

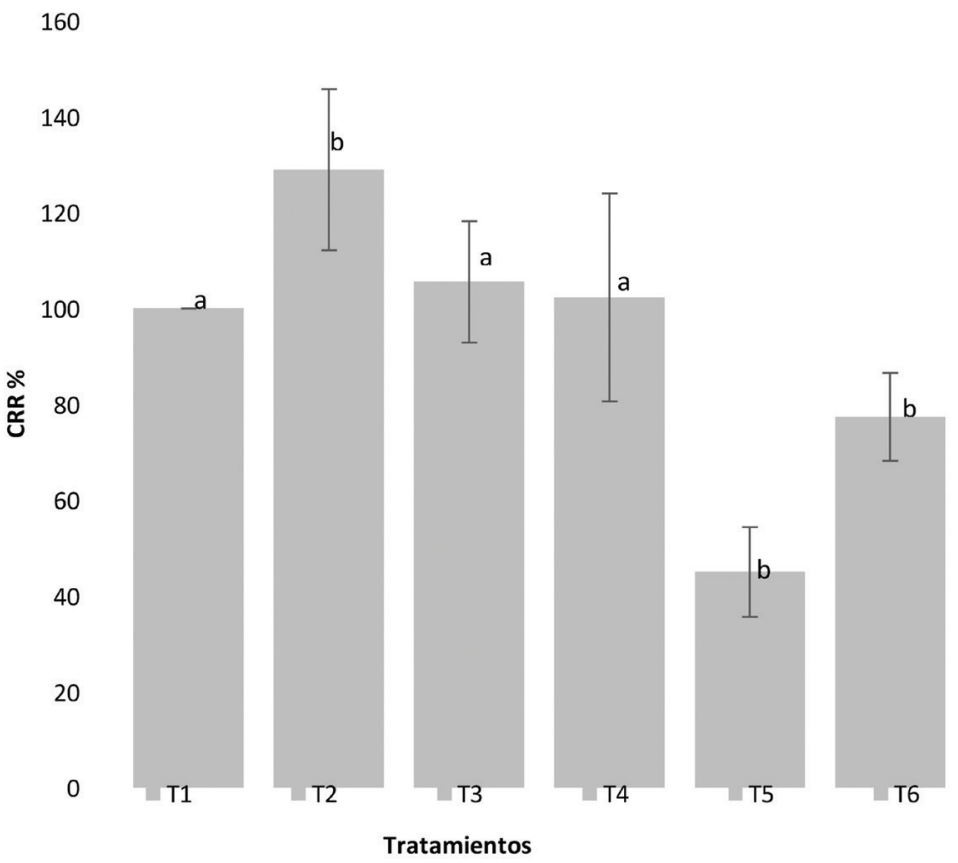

Fig. 2. Crecimiento Relativo Radicular (CRR\%) de semillas de pepino (Cucumis sativus) expuestas a T1 (compost puro sin MM o LDBIO), T2 (compost COMMR), T3 (compost COMMP), T4 (compost COLDBIO), T5 (compost COMMLDBIOA), T6 (compost COMMLDBIOB). Promedio de los bloques con sus respectivos errores estándar. (a) Tx= control ( $p>0,05),(b)$ Tx $\neq$ control $(p<0,05)$. Existen diferencias significativas entre el T2, T5 y T6 con respecto al control (Wilcoxon, $\mathrm{p}<0,05$ ). El empleo integrado de MM+LDBIO podría tener un efecto fitotóxico sobre cultivos sensibles. 


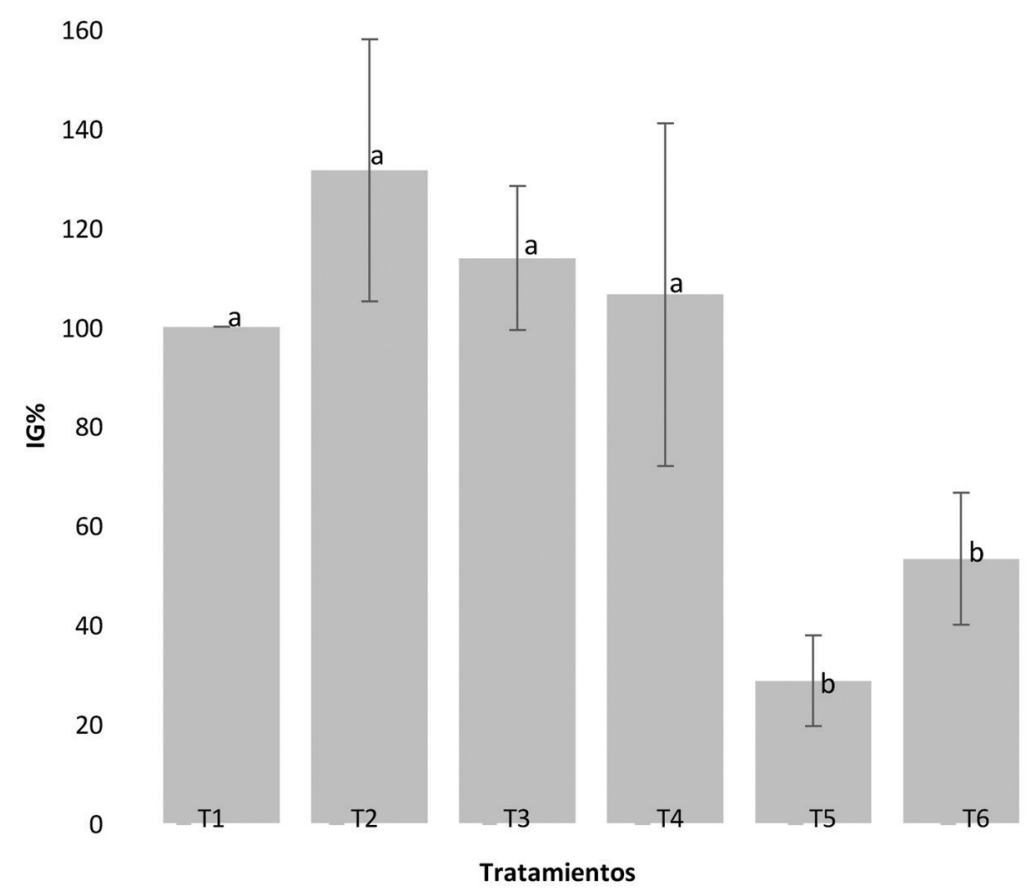

Fig. 3. Índice de Germinación (IG\%) de semillas de pepino (Cucumis sativus) expuestas a T1 (compost puro sin MM o LDBIO), T2 (compost COMMR), T3 (compost COMMP), T4 (compost COLDBIO), T5 (compost COMMLDBIOA), T6 (compost COMMLDBIOB).

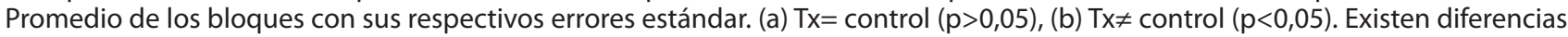
significativas entre el T5 y T6 con respecto al control (Wilcoxon, $\mathrm{p}<0,05$ ). El empleo integrado de MM+LDBIO podría tener un efecto fitotóxico sobre cultivos sensibles.

IG\% promedio de los tratamientos T5 y T6 es significativamente menor al control $(p<0,05)$. Estos promedios son menores al límite de fitotoxicidad propuestos por Zucconi (1981), y aportan evidencia de que el empleo integrado de microorganismos y lodos como agentes optimizadores del compost inhibe la germinación del cultivo de prueba, por lo que podría inferirse que la integración de estos dos compuestos podría generar efectos fitotóxicos en el compost maduro.

Índice de Crecimiento (IC): la prueba de Wilcoxon revela que el IC\% promedio de los tratamientos $\mathrm{T} 2$, T3 y T4 no difieren significativamente del control (T1) ( $p>0,05)$ (Fig. 4). Estos tratamientos presentan IC\% promedio cercanos al $100 \%$, por lo que según el criterio de Piemonte (2002), estos tres tipos de compost están libres de fitotoxinas. El IC\% promedio de los tratamientos T5 y T6 es significativamente menor al control $(p<0,05)$. Estos promedios son menores al límite de fitotoxicidad propuestos por Piemonte (2002), y aportan evidencia de que el empleo integrado de microorganismos y lodos como agentes optimizadores del compost inhibe el crecimiento del cultivo de prueba, por lo que podría inferirse que la integración de estos dos compuestos podría generar efectos fitotóxicos en el compost maduro.

\section{DISCUSIÓN}

Microorganismos de montaña y lodos digeridos de biodigestor como agentes optimizadores del compost: los microorganismos y los lodos son compuestos que han sido utilizados para diferentes aplicaciones en la agricultura. Ambos poseen atributos que los hacen ideales como agentes optimizadores del compost. Los microorganismos son abundantes, fáciles de conseguir, y tienen bajo costo de producción (aproximadamente US\$0,02/L). La inoculación sistemática de los residuos orgánicos durante el proceso de compostaje con microorganismos puede aumentar la tasa de descomposición del material compostable (Kausar et al., 2010; Amira et al., 2011; Mingyan, Xianlai, \& Xiaoqi, 2011; Parveen \& Padmaja, 2011; Wang, Fan, Hu, \& Yin, 2011; Hachicha et al., 2012; Saha et al., 2012), así como provocar una reducción del tiempo de maduración y un mejoramiento en la calidad final del compost (Wei et al., 2007), a través de la liberación de nutrientes disponibles para las plantas (Suchini 


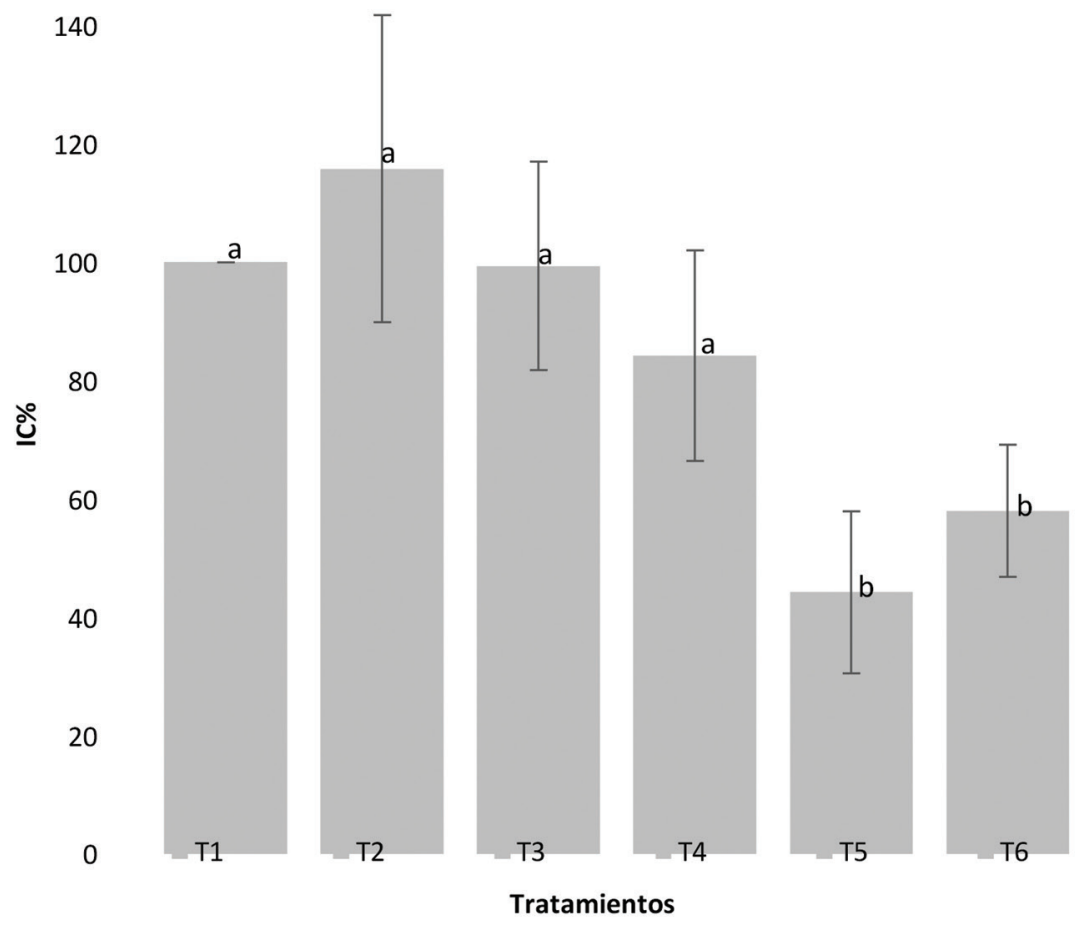

Fig. 4. Índice de Crecimiento (IC\%) de semillas de pepino (Cucumis sativus) expuestas a T1 (compost puro sin MM o LDBIO), T2 (compost COMMR), T3 (compost COMMP), T4 (compost COLDBIO), T5 (compost COMMLDBIOA), T6 (compost COMMLDBIOB). Promedio de los bloques con sus respectivos errores estándar. (a) Tx=control $(p>0,05),(b) T x \neq c o n t r o l(p<0,05)$. Existen diferencias significativas entre el T5 y T6 con respecto al control (Wilcoxon, p<0,05). El empleo integrado de MM+LDBIO podría tener un efecto fitotóxico sobre cultivos sensibles.

Ramírez, 2012). Su efectividad como biofertilizante ha sido comprobada en varios cultivos incluyendo tomate, acelga y cacao (Acosta Almánzar, 2012; Campo-Martínez, Acosta-Sanchez, Morales-Velasco, \& Prado 2014; Medina Flores, Loza, \& Augustín, 2014; Castro Barquero, Murillo Roos, Uribe Lorío, \& Mata 2015). La evaluación de la fitotoxicidad de los microorganismos hace un aporte en la validación de esta tecnología.

Los biosólidos resultantes de procesos de descontaminación anaeróbica como los lodos de biodigestor son abundantes, fáciles de conseguir y su empleo en la agricultura puede hacer un aporte en el contenido de nutrientes, materia orgánica y microorganismos en el agroecosistema (Mantovi, Baldoni, \& Toderi, 2005; Kinyua et al., 2016). Su recuperación es un mecanismo efectivo para el manejo integral de esta clase de residuos, lo cual es una práctica común en países industrializados y en desarrollo (Tsadilas, 2011; Larney \& Angers, 2012; Sharma, Sarkar, Singh, \& Singh, 2017). El manejo de los lodos puede ser complicado debido a los grandes volúmenes que se producen tanto a nivel doméstico como agroindustrial, así como por las características físicas y químicas del material (Sharma et al., 2017). El principal costo que conlleva su aprovechamiento es el transporte y procesamiento.

Los lodos pueden contener sustancias tóxicas como metales pesados por lo que es importante llevar a cabo procesos de evaluación de los niveles de toxicidad antes de utilizarlos como enmiendas agrícolas. La aplicación directa de los lodos puede generar problemas de lixiviación y contaminación del suelo y de las aguas superficiales y subterráneas (Clarke \& Cummins, 2015; Sharma et al., 2017), por lo que el compostaje es una alternativa más segura para el aprovechamiento de este material. Los lodos composteados son compuestos más estables (Mantovi et al., 2005) e inocuos (Sreesai, Peapueng, Tippayamongkonkun, \& Sthiannopkao, 2013) y su aplicación como biofertilizante no afecta las características físicas y microbiológicas del suelo (Sciubba et al., 2014; Joo, Monaco, Antmann, \& Chorath, 2015).

Fitotoxicidad de los microorganismos de montaña y los lodos digeridos de biodigestor: el bioensayo de fitotoxicidad revela que la aplicación de microorganismos y lodos como agentes optimizadores del compost 
en forma individual no produce efectos inhibidores de la germinación ni el crecimiento del cultivo de prueba, por lo que es razonable afirmar que, de acuerdo al sistema producción descrito, estos dos compuestos están libres de fitotoxinas y son seguros para los cultivos. Los resultados también muestran que el empleo integrado de ambos agentes ( $M M+L D B I O)$, tiene efectos inhibidores sobre la germinación y el crecimiento del cultivo de prueba, lo cual indica que podrían haber compuestos tóxicos que reaccionan durante el proceso de compostaje que podrían provocar problemas en la germinación y el desarrollo de los cultivos.

Para poder dilucidar las causas de la fitotoxicidad del compost cuando se aplica microorganismos y lodos como agentes optimizadores en forma integrada, es necesario llevar a cabo pruebas más extensivas a nivel químico. Sin embargo, una explicación razonable podría estar ligada al exceso de Zinc (Zn) presente en el compost que contiene lodos, lo cual según Stoffella et al. (2001), puede provocar inhibición en la germinación y el crecimiento de cultivos sensibles cuando las concentraciones sobrepasan Zn 25,0mg. $\mathrm{kg}^{-1}$.

La concentración inicial de $\mathrm{Zn}$ fue de 1 129,0 $\mathrm{mg}^{*} \mathrm{~kg}^{-1}$ en el lodo sin compostear y de $2,0 \mathrm{mg}^{*} \mathrm{~kg}^{-1}$ en el cultivo de microorganismos, mientras que la concentración promedio de este elemento fue de $112,3 \mathrm{mg}^{*} \mathrm{~kg}^{-1}$ en los compost $\sin$ lodos, en contraste a $145,6 \mathrm{mg}^{\mathrm{kg}} \mathrm{kg}^{-1}$ en los compost con lodos (Camacho-Céspedes et al., 2018) ( 30\% de diferencia). En ambos casos, la concentración de este microelemento sobrepasa en más del 100\% el umbral de fitotoxicidad reportado por Stoffella et al. (2001), lo cual podría explicar el efecto inhibitorio observado sobre la germinación y crecimiento en el ensayo experimental.

El origen del exceso de Zn podría explicarse por el hecho de que la alimentación de los cerdos que aportan las excretas para la formación de los lodos empleados para el presente estudio, es suplementada en aproximadamente $50 \%$ de la dieta diaria con concentrados preparados agroindustrialmente, los cuales son suplementados con Zn para contrarrestar la toxicidad del Cu (Tam \& Tiquia, 1994). Estos elementos son absorbidos metabólicamente en bajas cantidades (5-10\%) por lo que el resto es desechado a través de las excretas (Pampuro et al., 2017).

El exceso de B es otro factor que podría causar inhibición en la germinación y el crecimiento de los cultivos cuando las concentraciones sobrepasan los B 2,5 $\mathrm{mg}^{*} \mathrm{~kg}^{-1}$ (Stoffella et al., 2001). El cultivo de microorganismos registra una concentración inicial de $1,0 \mathrm{mg}^{*} \mathrm{~kg}^{-1}$ mientras que en los lodos la cantidad reportada es de $10,0 \mathrm{mg}^{*} \mathrm{~kg}^{-1}$ (Camacho-Céspedes et al., 2018). El promedio de la concentración de este micronutriente en los compost tratados con lodos es $\sim 10 \%$ mayor a los compost que están libres de este biosólido. En ambos casos la concentración de este elemento es más del 100\% del umbral máximo recomendado por Stoffella et al. (2001), por lo que el exceso de este micronutriente podría ser otra causa de la fitotoxicidad detectada en el compost enmendado con microorganismos y lodos.

Bernal et al. (2017) reportan que una de las principales causas de la fitotoxicidad del compost es la presencia de sales en el sustrato, lo cual puede ser detectado a través de la lectura de la conductividad eléctrica. Este parámetro debe permanecer en $\sim 10,0 \mathrm{mS} / \mathrm{cm}$ según US Composting Council (2002) para evitar que haya problemas de fitotoxicidad. Las puntuaciones para esa variable en el caso de los microorganismos fueron de CE $7,5 \mathrm{mS} / \mathrm{cm}$, mientras que en los lodos alcanzó $6,6 \mathrm{mS} / \mathrm{cm}$ (Camacho-Céspedes et al., 2018). En el caso de los compost que no contienen biosólidos, la CE promedio registró $9,6 \mathrm{mS} / \mathrm{cm}$ mientras que el que sí contiene ambos agentes optimizadores, la CE alcanzó $9,5 \mathrm{mS} / \mathrm{cm}$, por lo que se descarta que el exceso de sales, utilizando el parámetro de CE como proxi, sea el factor que esté causando la fitotoxicidad detectada.

Utilizando los PRSG\%, CCR\%, IG\%, IC\% como parámetros discriminatorios de fitotoxicidad, se concluye que el empleo individual de microorganismos de montaña y lodos digeridos de biodigestor como agentes optimizadores del compost en las concentraciones utilizadas, no tiene un efecto inhibidor en la germinación y el crecimiento del cultivo de prueba (Cucumis sativus), y se infiere por deducción que la utilización individual de estos compuestos no produce un efecto fitotóxico sobre cultivos sensibles, por lo que es seguro, desde el punto de vista de fitotoxicidad, emplearlos como agentes optimizadores del compost en forma separada. La incorporación simultánea de ambos agentes en la producción de compost provocó una disminución significativa en la germinación y el crecimiento inicial del cultivo de prueba. Este resultado podría estar relacionado al efecto fitotóxico provocado por el exceso de Zinc y Boro detectado en el compost que combina ambos compuestos. Se recomienda utilizar este compost con precaución y llevar a cabo otras pruebas de crecimiento para identificar si la fitotoxicidad observada se mantiene sobre otros cultivos y sobre otras etapas de desarrollo más avanzadas a la germinación y el crecimiento inicial.

\section{AGRADECIMIENTOS}

Los autores agradecen a University of Georgia Costa Rica Campus y al Concejo Nacional para Investigaciones 
Tecnológicas de Costa Rica por el apoyo logístico y económico brindado para la elaboración del presente estudio.

\section{REFERENCIAS}

Acosta Almánzar, H.A. (2012). Microorganismos eficientes de montaña: evaluación de su potencial bajo manejo agroecológico de tomate en Costa Rica. Turrialba, Costa Rica: CATIE.

Amira, R.D., Roshanida, A., Rosli, M., Zahrah, M.S.F., Anuar, J.M., \& Adha, C.N. (2011). Bioconversion of empty fruit bunches (EFB) and palm oil mill effluent (POME) into compost using Trichoderma virens. African Journal of Biotechnology, 10(81), 18775-18780.

Beltrán, E., Miralles de Imperial, R., Porcel, M., Delgado, M., Beringola, M., Martín, J., \& Bigeriego, M. (2002). Effect of sewage sludge compost application on ammonium-nitrogen and nitrate-nitrogen contents of an olive grove soil. Revista internacional de contaminación ambiental, 21(3), 143-150.

Bernal, M.P., Sommer, S.G., Chadwick, D., Qing, C., Guoxue, L., \& Michel Jr, F.C. (2017). Current Approaches and Future Trends in Compost Quality Criteria for Agronomic, Environmental, and Human Health Benefits. Advances in agronomy, 144, 143-233. DOI: 10.1016/ bs.agron.2017.03.002

Boldrin, A., Andersen, J.K., Møller, J., Christensen, T.H., \& Favoino, E. (2009). Composting and compost utilization: accounting of greenhouse gases and global warming contributions. Waste Management \& Research, 27(8), 800-812. DOI: $10.1177 / 0734242 X 09345275$

Bot, A., \& Benites, J. (2005). The Importance of Soil Organic Matter: Key to Drought-resistant Soil and Sustained Food Production. Rome, Italy: Food and Agriculture Organization of the United Nations.

Camacho-Céspedes, F., Uribe-Lorío, L., Newcomer, Q., Masters, K.L., \& Kinyua, M. (2018). Bio-optimización del compost con cultivos de microorganismos de montaña (MM) y lodos digeridos de biodigestor (LDBIO). UNED Research Journal, 10(2), 330-341. DOI: 10.22458/urj.v10i2.2163

Campitelli, P., \& Ceppi, S. (2008). Chemical, physical and biological compost and vermicompost characterization: A chemometric study. Chemometrics and Intelligent Laboratory Systems, 90(1), 64-71. DOI: 10.1016/j. chemolab.2007.08.001

Campo-Martínez, A., Acosta-Sanchez, R.L., Morales-Velasco, S., \& Prado, F.A. (2014). Evaluación de microorganismos de montaña $(\mathrm{mm})$ en la producción de acelga en la meseta de Popayán. Biotecnología en el Sector Agropecuario y Agroindustrial, 12(1), 79-87.

Castro Barquero, L., Murillo Roos, M., Uribe Lorío, L., \& Mata Chinchilla, R. (2015). Inoculación al suelo con Pseudomonas fluorescens, Azospirillum oryzae, Bacillus subtilis y microorganismos de montaña $(\mathrm{mm})$ y su efecto sobre un sistema de rotación soya-tomate bajo condiciones de invernadero. Agronomía Costarricense, 39(3), 21-36.

Clarke, R.M., \& Cummins, E. (2015). Evaluation of "classic" and emerging contaminants resulting from the application of biosolids to agricultural lands: A review. Human and Ecological Risk Assessment: An International Journal, 21(2), 492-513. DOI: 10.1080/10807039.2014.930295

Cohen, S., Flint, M.L., \& Hines, N. (2009). Lawn and Residential Landscape Pest Control: A Guide for Maintenance Gardeners. California, U.S.A.: University of California, Statewide Integrated Pest Management Program, Agriculture and Natural Resources.

Diaz, L.F., De Bertoldi, M., \& Bidlingmaier, W. (Eds.). (2011). Compost science and technology (Vol. 8, $1^{\text {st }}$ ed.). Oxford, U. K.: Elsevier Science.

FAO (Food and Agriculture Organization of the United Nations). (2013). Manual de Compostaje del Agricultor. Santiago de Chile: FAO.

Golabi, M.H., Denney, M., \& lyekar, C. (2007). Value of composted organic wastes as an alternative to synthetic fertilizers for soil quality improvement and increased yield. Compost science \& utilization, 15(4), 267-271. DOI: 10.1080/1065657X.2007.10702343

Hachicha, R., Rekik, O., Hachicha, S., Ferchichi, M., Woodward, S., Moncef, N., . . Mechichi, T. (2012). Co-composting of spent coffee ground with olive mill wastewater sludge and poultry manure and effect of Trametes versicolor inoculation on the compost maturity. Chemosphere, 88(6), 677-682. DOI: 10.1016/j.chemosphere.2012.03.053

Haug, R.T. (1993). The practical handbook of compost engineering. Florida, U.S.A.: CRC Press.

Helfrich, P., Chefetz, B., Hadar, Y., Chen, Y., \& Schnabl, H. (1998). A novel method for determining phytotoxicity in composts. Compost Science \& Utilization, 6(3), 6-13. DOI: 10.1080/1065657X.1998.10701926

Hernández Sampieri, R., Fernández Collado, C., \& Baptista Lucio, P. (2010). Metodología de la investigación. México: Editorial Mc Graw Hill.

Joo, S.H., Monaco, F.D., Antmann, E., \& Chorath, P. (2015). Sustainable approaches for minimizing biosolids production and maximizing reuse options in sludge management: A review.Journalofenvironmentalmanagement, 158, 133-145. DOI: 10.1016/j.jenvman.2015.05.014

Kausar, H., Sariah, M., Saud, H.M., Alam, M.Z., \& Ismail, M.R. (2010). Development of compatible lignocellulolytic fungal consortium for rapid composting of rice straw. International Biodeterioration \& Biodegradation, 64(7), 594-600. DOI: 10.1016/j.ibiod.2010.06.012

Kinyua, M., Zhang, J., Camacho-Céspedes, F., Tejada-Martinez, A., \& Ergas, S. (2016). Use of physical and biological process models to understand the performance of tubular anaerobic digesters. Biochemical Engineering Journal, 107, 35-44. DOI: 10.1016/j.bej.2015.11.017

Kotschi, J. (2015). A Soiled reputation. Adverse impacts of mineral fertilizers in tropical agriculture. Germany:WWFGermanyHeinrich Böll Stiftung. 
Larney, F.J., \& Angers, D.A. (2012). The role of organic amendments in soil reclamation: A review. Canadian Journal of Soil Science, 92(1), 19-38. DOI: 10.4141/cjss2010-064

Lazcano, C., Martínez-Blanco, J., Christensen, T.H., Muñoz, P., Rieradevall, J., Møller, J., ... Nuñez, M. (2014). Environmental benefits of compost use on land through LCA - a review of the current gaps. In R. Schenck, \& D. Huizenga (Eds.), Proceedings of the 9th International Conference on Life Cycle Assessment in the Agri-Food Sector (pp. 674-682). San Francisco, California, U.S.A.: ACLCA.

Lehner, P., \& Rosenberg, N.A. (2017). Legal Pathways to CarbonNeutral Agriculture. News and Analysis Environmental Law Reporter, 10(47), 10845-10876.

Mantovi, P., Baldoni, G., \& Toderi, G. (2005). Reuse of liquid, dewatered, and composted sewage sludge on agricultural land: effects of long-term application on soil and crop. Water research, 39(2), 289-296. DOI: 10.1016/j. watres.2004.10.003

Medina Flores, C.M., Loza, T., \& Augustín, J. (2014). Efecto de dosis y aplicaciones edáficas y foliar de microorganismos de montaña con y sin sales minerales en el rendimiento del cacao (Theobroma cacao l.) variedad criolla, municipio San José de Bocay, Jinotega, febrero-mayo del 2014 (tesis Ingeniero en Agroecología Tropical). Universidad Nacional Autónoma de Nicaragua, León, Nicaragua.

Mingyan, Y., Xianlai, Z., \& Xiaoqi, Z. (2011). Screening of complex thermophilic microbial community and application during municipal solid waste aerobic composting. African Journal of Biotechnology, 10(67), 15163-15169. DOI: 10.5897/AJB10.2559

Pampuro, N., Bisaglia, C., Romano, E., Brambilla, M., Foppa Pedretti, E., \& Cavallo, E. (2017). Phytotoxicity and Chemical Characterization of Compost Derived from Pig Slurry Solid Fraction for Organic Pellet Production. Agriculture, 7(11), 94. DOI: 10.3390/agriculture7110094

Parveen, A.A., \& Padmaja, C. (2011). Efficacy of fungi and actinomycetes in converting municipal solid waste (MSW) and water hyacinth $(\mathrm{WH})$ into organic manure. Reserach on crops, 12(1), 167-172.

Paulin, B., \& O'Malley, P. (2008). Compost production and use in horticulture. Western Australia: Western Australia Agriculture Authority.

Piemonte, R. (2002). Il compostaggio. Processo, tecniche e applicazione. Torino, Italia: Collana Ambiente.

R Core Team. (2018). R: A Language and Environment for Statistical Computing. Vienna, Austria: R Foundation for Statistical Computing.

Reigosa, M., \& Pazos-Malvido, E. (2007). Phytotoxic effects of 21 plant secondary metabolites on Arabidopsis thaliana germination and root growth. Journal of Chemical Ecology, 33(7), 1456-1466. DOI: 10.1007/ s10886-007-9318-x

Saha, N., Mukherjee, D., Sen, S., Sarkar, A., Bhattacharaya, K., Mukhopadyay, N., \& Patra, P. (2012). Application of highly efficient lignocellulolytic fungi in cocomposting of paddy straw amended poultry droppings for the production of humus rich compost. Compost Science \& Utilization, 20(4), 239-244. DOI: 10.1080/1065657X.2012.10737054

Sciubba, L., Cavani, L., Negroni, A., Zanaroli, G., Fava, F., Ciavatta, C., \& Marzadori, C. (2014). Changes in the functional properties of a sandy loam soil amended with biosolids at different application rates. Geoderma, 221, 40-49. DOI: 10.1016/j.geoderma.2014.01.018

Sharma, B., Sarkar, A., Singh, P., \& Singh, R.P. (2017). Agricultural utilization of biosolids: A review on potential effects on soil and plant grown. Waste Management, 64, 117-132. DOI: 10.1016/j.wasman.2017.03.002

Sreesai, S., Peapueng, P., Tippayamongkonkun, T., \& Sthiannopkao, S. (2013). Assessment of a potential agricultural application of Bangkok-digested sewage sludge and finished compost products. Waste Management \& Research, 31(9), 925-936. DOI: $10.1177 / 0734242 \times 13494261$

Stoffella, P.J., \& Kahn, B.A. (2001). Compost utilization in horticultural cropping systems. Florida, U.S.A.: CRC Press. DOI: $10.1201 / 9781420026221$

Suchini Ramírez, J.G. (2012). Innovaciones agroecológicas para una producción agropecuaria sostenible en la región del Trifinio. Turrialba, Costa Rica: CATIE.

Szabó, L.G. (2000). Juglone index: A possibility for expressing allelopathic potential of plant taxa with various life strategies. Acta Botanica Hungarica, 42(1-4), 295-305.

Tam, N., \& Tiquia, S. (1994). Assessing toxicity of spent pig litter using a seed germination technique. Resources, Conservation and Recycling, 11(1-4), 261-274. DOI: 10.1016/0921-3449(94)90094-9

Tsadilas, C.D. (2011). Heavy Metals Forms in Biosolids, Soils, and Biosolid-Amended Soils. In H. M. Selim (Ed.), Dynamics and Bioavailability of Heavy Metals in the Rootzone (pp. 277-297). Florida, U.S.A.: CRC Press. DOI: 10.1201/b10796

US Composting Council. (2002). Test methods for the examination of composting and compost. Reston, Vermont, U.S.A.: US Composting Council.

Wang, H.Y., Fan, B.Q., Hu, Q.X., \& Yin, Z.W. (2011). Effect of inoculation with Penicillium expansum on the microbial community and maturity of compost. Bioresource technology, 102(24), 11189-11193. DOI: 10.1016/j. biortech.2011.07.044

Wang, W., \& Keturi, P.H. (1990). Comparative seed germination tests using ten plant species for toxicity assessment of a metal engraving effluent sample. Water, Air, and Soil Pollution, 52(3-4), 369-376.

Wei, Z., Xi, B., Zhao, Y., Wang, S., Liu, H., \& Jiang, Y. (2007). Effect of inoculating microbes in municipal solid waste composting on characteristics of humic acid. Chemosphere, 68(2), 368-374. DOI: 10.1016/j.chemosphere.2006.12.067

Zucconi, F. (1981). Evaluating toxicity of immature compost. BioCycle, 22(2), 54-57. 